\title{
NMA OBSERVATIONS OF HH83
}

\author{
MAKOTO NAKANO \\ Oita University, Oita 870-11, Japan
}

KOJI SUGITANI

Nagoya City University, Mizuho-ku, Nagoya 467, Japan

FUMIO SATO

Tokyo Gakugei University, Koganei, Tokyo 184, Japan

KATSUO OGURA

Kokugakuin University, Shibuya-ku, Tokyo 150, Japan

ABSTRACT We have reported NMA observations of HH83 with CS $(\mathrm{J}=2-1)$ line. The interacton between the outflow and the molecluar cloud is found.

\section{INTRODUCTION}

HH 83 was discovered independently by Reipurth and Graham (1988) and Ogura and Walsh (1991) near the western edge of L1641 in Orion. It has many optical features typical of Herbig-Haro (HH) Objects like HH34 and L1551 IRS5. The driving source is identified with IRAS 05311-0631 with a luminosity of $10 L_{\odot}$, which is considered to be an embedded $T$ Tauri star with active disk from its spectral energy distribution and the optical spectrum of the reflection nebula.

\section{OBSERVATIONS AND RESULTS}

We have observed HH83 with the Nobeyama Millimeter Array (NMA) in the line of CS $(\mathrm{J}=2-1)$. The frontend were SIS receivers, and the backend was a digital FFT spectrometer with a total bandwidth of $320 \mathrm{MHz}$. The velocity resolution was $0.96 \mathrm{~km} / \mathrm{s}$. The $\mathrm{D}$ configurations of $\mathrm{NMA}$ were employed and the synthesized beam size was $15.3^{\prime \prime} \times 6.2^{\prime \prime}$. We have also observed CS $(\mathrm{J}=2-1)$, CO $(\mathrm{J}=1-0)$, and ${ }^{13} \mathrm{CO}(\mathrm{J}=1-0)$ lines with the Nobeyama $45-\mathrm{m}$ telescope.

Our abservations of HH83 show that the main CS cloud, which was found by using the 45-m telescope, is elongated in SE-NW direction, and composed of at least two compact clumps ( SE and NW clump ) with 10" separation. The size of clumps is about $5000 \mathrm{AU}$ each. By assuming that the CS $(\mathrm{J}=2-1)$ line is optically thin, and abundance ratio $\mathrm{X}(\mathrm{CS})=10^{-9}$, the mass of each clump is estimated to be about $0.5 M_{\odot}$.

The peak of the SE clump coincides with the driving source HH83IRS ( RA $=5^{h} 31^{m} 06.2^{s}$, Dec. $\left.=-6^{\circ} 31^{\prime} 44^{\prime \prime}\right)^{1}$ which corresponds to the IRAS source. 


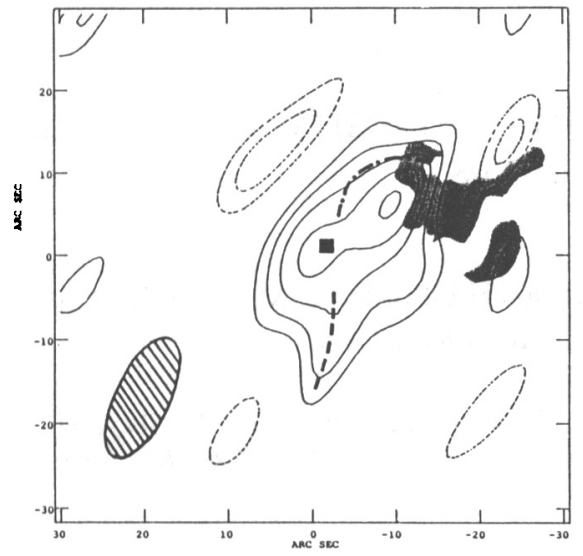

FIGURE I Contour map of CS J=2-1 emission at the center velocity of 6.4 $\mathrm{km} / \mathrm{s}$ with $0.96 \mathrm{~km} / \mathrm{s}$ width. Contour levels start at $330 \mathrm{mJy} / \mathrm{beam}$ in steps of $165 \mathrm{mJy} /$ beam ( $\mathrm{rms}=165 \mathrm{mJy} /$ beam ). Negative contours are shown in dashed line. An deep [SII] image of HH83 by Reipurth (1989) is illustrated by shading and HH83IRS is indicated by the solid square. The dashed line shows the southern CS ridge, and the dash-dotted line shows the ridge structure, which was found on our near infrared image.

This clump seems to be the remnant of the parental cloud which formed it. The feature extending from HH83IRS to the south is also found. It may represent the molecular ridge along the SE cavity wall, which is associated with the counter flow. The NW clump is outlined by an arc structure of the knot B and C, which are characterized by their $\mathrm{HH}$ emission and the reflected star light, on the western side and infrared nebulosity ${ }^{1}$ on the other side. This appearance suggests that the outflow from HH83IRS has interacted with the NW clump, and the $\mathrm{HH}$ emission is formed at the shocked interface region.

The blue and the red component of the CS cloud are displaced each other. The blue one seems to be elogated to the assumed northern edge of the NW cavity wall. The weak CS emission associated with knot $\mathrm{A}$ has also been found. We found HH emission nebulosity near the root of the optical jet lies inside of the CS emission. We presumably found the site of interaction of the outflow with the ambient molecular cloud.

\section{REFERENCES}

Ogura,K., and Walsh,J.R. 1991, $A J$, 101,185

Reipurth,B., and Graham,J.A. 1988, $A \cup A, 202,219$

Reipurth,B. 1989, $A B A, 220,249$

\footnotetext{
${ }^{1}$ We have obtained the near infrared images of HH83 by using the $1.5-\mathrm{m}$ telescope at the Communication Research Laboratory with an HgCdTe $128 \times 128$ array camera. The position of HH83IRS was measured on these images within an uncertainty of 1 ".
} 